\title{
Cholinergic Modulation of Vibrissal Receptive Fields in Trigeminal Nuclei
}

\author{
Elena Timofeeva, Caroline Dufresne, Attila Sík, Zhong-Wei Zhang, and Martin Deschênes \\ Centre de Recherche Université Laval-Robert Giffard, Québec City, Québec, Canada G1J 2G3
}

In sensory systems, it is usually considered that mesopontine cholinergic neurons exert their modulatory action in the thalamus by enhancing the relay of sensory messages during states of neural network desynchronization. Here, we report a projection heretofore unknown of these cholinergic cells to the interpolar division of the brainstem trigeminal complex in rats. After FluoroGold injection in the interpolar nucleus, a number of retrogradely labeled cells were found bilaterally in the pedunculopontine tegmental nucleus, and immunostaining revealed that the vast majority of these cells were also positive for choline acetyltransferase. Immunostaining for the acetylcholine vesicular transporter confirmed the presence of cholinergic terminals in the interpolar nucleus, where electron microscopy showed that they make symmetric and asymmetric synaptic contacts with dendrites and axon terminals. In agreement with these anatomical data, recordings in slices showed that the cholinergic agonist carbachol depolarizes large-sized interpolaris cells and increases their excitability. Local application of carbachol in vivo enhances responses to adjacent whiskers, whereas systemic administration of the cholinergic antagonist scopolamine produces an opposite effect. Together, these results show that mesopontine cholinergic neurons exert a direct, effective control over receptive field size at the very first relay stations of the vibrissal system in rodents. As far as receptive field synthesis in the lemniscal pathway relies on intersubnuclear projections from the spinal complex, it follows that cholinergic modulation of sensory transmission in the interpolar nucleus will have a direct bearing on the type of messages that is forwarded to the thalamus and cerebral cortex.

Key words: barrels; whisker; receptive field; trigeminal nuclei; acetylcholine; reticular formation; arousal

\section{Introduction}

The brainstem trigeminal complex is the first relay station in the vibrissal system of rodents. From there, two pathways convey vibrissal information to the cerebral cortex: (1) the lemniscal pathway, which arises from the principal trigeminal nucleus $(\mathrm{PrV})$, transits through the ventral posterior medial nucleus (VPM) of the thalamus, and terminates in the granular zone of the cortical barrel field; (2) the paralemniscal pathway, which arises from the interpolar division of the spinal trigeminal complex (SpVi), transits through the posterior group nucleus, and terminates in the dysgranular zone of the cortical barrel field. In the brainstem, lemniscal and paralemniscal streams of information processing are not completely isolated from each other, in that the PrV receives abundant projections from the spinal complex (Jacquin et al., 1990a; Timofeeva et al., 2004).

Primary vibrissa afferents form ladder-like projection patterns throughout the trigeminal column, where they give off several clusters of terminations that are spatially restricted to homotopic whisker-related modules called barrelettes (Henderson and Jacquin, 1995). Neurons in the PrV that give rise to the lemniscal

Received Jan. 19, 2005; revised Aug. 22, 2005; accepted Aug. 22, 2005.

This work was supported by Canadian Institutes for Health Research grants to M.D., Z.-W.Z., and A.S. We thank Philippe Lavallée for his contribution in figure editing.

Correspondence should be addressed to Martin Deschênes, Centre de Recherche Université Laval-Robert Giffard, 2601 de la Canardière, Québec City, Québec, Canada G1J 2G3. E-mail: martin.deschenes@crulrg.ulaval.ca. DOI:10.1523/JNEUROSCI.3073-05.2005

Copyright $\odot 2005$ Society for Neuroscience $\quad$ 0270-6474/05/259135-09\$15.00/0 pathway have dendrites confined within the limit of their home barrelette and thus have receptive fields dominated by a single whisker (Henderson and Jacquin, 1995; Veinante and Deschênes, 1999). In contrast, thalamic projecting interpolaris neurons have dendritic trees that extend across several barrelettes, and they respond vigorously to the deflection of several whiskers (Woolston et al., 1982; Jacquin et al., 1989b; Veinante et al., 2000). In lightly anesthetized animals, the multiwhisker responses of SpVi neurons are effectively relayed to the PrV by intersubnuclear axons, which renders PrV and VPM cells responsive to several adjacent whiskers (Minnery and Simons, 2003; Timofeeva et al., 2004). Lesion studies in which ascending intersubnuclear axons were destroyed provided clear evidence that adjacent whisker responses in PrV, VPM, and barrel cortex rely primarily on projections from the spinal trigeminal complex to the PrV (Rhoades et al., 1987; Friedberg et al., 1999; Timofeeva et al., 2004; Kwegyir-Afful et al., 2005). In one of these studies (Timofeeva et al., 2004), it was also reported that a midline brainstem lesion at the pontine level severely reduced the receptive field size of interpolaris cells. That result was intriguing, because it suggested that brainstem modulatory systems might control tactile responsiveness in the SpVi. The present study aimed at establishing which modulatory system(s) might be involved.

\section{Materials and Methods}

In vivo experiments were performed in male rats (Sprague Dawley; 250$300 \mathrm{~g}$ ) in accordance with federally prescribed animal care and use guide- 
lines. The University Committee for Animal Use in Research approved all experimental protocols.

Tract tracing experiments. Four rats were anesthetized with ketamine ( $75 \mathrm{mg} / \mathrm{kg}$ ) plus xylazine $(5 \mathrm{mg} / \mathrm{kg}$ ), and the whisker-responsive region of the SpVi was identified by single-unit recordings. Then, a micropipette (tip diameter, $20 \mu \mathrm{m})$ containing FluoroGold (FG) $(2 \%$ in $0.1 \mathrm{M} \mathrm{caco-}$ dylate buffer, $\mathrm{pH} 7.0$ ) was lowered in that same region, and the tracer was ejected with positive current pulses (on/off duration, $2 \mathrm{~s}$ ) of $600 \mathrm{nA}$ for 20 min. After tracer injection, the skin was sutured, rats were given analgesics (Anafen; $5 \mathrm{mg} / \mathrm{kg}$ ), and they were returned to the animal facilities. After a survival period of $48 \mathrm{~h}$, rats were perfused under ketaminexylazine anesthesia with a solution of $4 \%$ paraformaldehyde and $0.5 \%$ glutaraldehyde in PBS (0.1 M; pH 7.4). Brains were removed, cryoprotected in $30 \%$ sucrose overnight, and brainstems were cut coronally at 70 $\mu \mathrm{m}$ on a freezing microtome. The cortices were not processed for analyses. Sections were serially collected in PBS, and alternate sections were processed for cytochrome oxidase histochemistry and FG immunochemistry. Before immunostaining, sections were treated for $30 \mathrm{~min}$ with a solution of $50 \%$ ethanol plus $1 \%$ hydrogen peroxide. After several rinses in PBS, sections were preincubated for $1 \mathrm{~h}$ in PBS with 3\% normal goat serum and $0.3 \%$ Triton $\mathrm{X}-100$. They were incubated overnight in the same medium containing an anti-FG antiserum (1:8000 dilution; rabbit polyclonal IgG; Chemicon, Temecula, CA). Then, sections were rinsed in PBS and incubated for $1 \mathrm{~h}$ with a mixture of normal goat serum (3\%), Triton X-100 (0.3\%), and biotinylated goat anti-rabbit IgG (1:200 dilution; Vector Laboratories, Burlingame, CA). Finally they were incubated with an avidin-biotin peroxidase complex (Vectastain ABC Elite kit; Vector Laboratories), and revealed in Tris- $\mathrm{HCl}$ buffer, $\mathrm{pH}$ 7.6, containing the chromagen 3,3'-diaminobenzidine tetrahydrochloride (DAB) $(0.05 \%)$, nickel ammonium sulfate $(0.05 \%)$, and $0.001 \%$ hydrogen peroxide.

Retrograde tracing and choline acetyltransferase immunochemistry. In 3 rats FG was injected in the SpVi as described above, and coronal brainstem sections $(50 \mu \mathrm{m})$ were processed for choline acetyltransferase (ChAT) immunohistochemistry. After preincubation for $1 \mathrm{~h}$ in PBS with $3 \%$ normal goat serum and $0.3 \%$ Triton X-100, sections were incubated overnight in the same mixture containing ChAT antibody (1:200 dilution; mouse monoclonal IgG; Chemicon). A secondary antibody conjugated to Alexa Fluor 568 (1:200 dilution; goat anti-mouse IgG; Invitrogen, Eugene, OR) was used to reveal immunostaining.

Vesicular acetylcholine transporter immunochemistry. Cholinergic terminals were visualized by vesicular acetylcholine transporter (VAChT) immunostaining using rabbit polyclonal antibodies directed against the VAChT C terminus (generous gift from Dr. R. H. Edwards, University of California, San Francisco, CA). In two experiments, the SpVi was outlined by retrograde labeling after FG injection in the whisker-responsive region of the $\mathrm{PrV}$, and immunostaining was performed as described above using VAChT antibody (1:5000 dilution), which was visualized with a secondary antibody conjugated to Alexa Fluor 568 (1:200 dilution; goat anti-rabbit IgG; Invitrogen).

Electron microscopy. One rat was perfused under deep ketamine-xylazine anesthesia, and horizontal sections ( $50 \mu \mathrm{m}$ thick) of the brainstem were cut on a vibratome. After several rinses in phosphate buffer (PB), sections were cryoprotected in $30 \%$ sucrose in $0.1 \mathrm{M} \mathrm{PB}$ and freezethawed in an aluminum-foil boat over liquid nitrogen. Sections were incubated with the VAChT antibody as described above (without Triton $\mathrm{X}-100$ ), and immunostaining was revealed with a biotinylated goat antirabbit IgG (1:200), the ABC complex, and nickel-DAB. After osmication ( $1 \%$ osmium tetroxide in $\mathrm{PB}$ for $30 \mathrm{~min}$ ), sections were washed in $\mathrm{PB}$, dehydrated in a graded series of ethanol (with $1 \%$ uranyl acetate in $70 \%$ ethanol), cleared in propylene oxide, and flat embedded in Durcupan. Thin sections were serially cut on an ultramicrotome, collected on formvar-coated single-slot grids, stained with lead citrate, and examined with a Philips Tecnai 12 EM.

Patch-clamp recording in slices. Brain slices were prepared from Sprague Dawley rats of either sex, aged postnatal day 14 (P14) to P18 (with the day of birth as $\mathrm{P} 0$ ). Rats were deeply anesthetized with ketamine and xylazine, and decapitated. The brain was removed quickly ( $<60 \mathrm{~s})$ and placed in an ice-cold solution containing the following (in $\mathrm{mm}$ ): 210 sucrose, $3.0 \mathrm{KCl}, 1.0 \mathrm{CaCl}_{2}, 3.0 \mathrm{MgSO}_{4}, 1.0 \mathrm{NaH}_{2} \mathrm{PO}_{4}, 26 \mathrm{NaHCO}_{3}, 10$ glucose, saturated with $95 \% \mathrm{O}_{2}$ and $5 \% \mathrm{CO}_{2}$. Horizontal slices of the brainstem were cut at $250 \mu \mathrm{m}$ on a vibrating tissue slicer (VT 1000s; Leica, Nussloch, Germany) and kept in artificial CSF (ACSF) containing the following (in mM): $124 \mathrm{NaCl}, 3.0 \mathrm{KCl}, 1.5 \mathrm{CaCl}_{2}, 1.3 \mathrm{MgCl}_{2}, 1.0$ $\mathrm{NaH}_{2} \mathrm{PO}_{4}, 26 \mathrm{NaHCO}_{3}, 20$ glucose, saturated with $95 \% \mathrm{O}_{2}$ and $5 \% \mathrm{CO}_{2}$ at room temperature. Slices were allowed to recover for at least $1 \mathrm{~h}$ before recording.

For recording, a slice was transferred to a submerge-type chamber, in which it was continuously exposed to ACSF, saturated with $95 \% \mathrm{O}_{2}$ and $5 \% \mathrm{CO}_{2}$ (flow rate, $2.0 \pm 0.2 \mathrm{ml} / \mathrm{min}$ ). The slice was viewed first with a $4 \times$ objective, and the SpVi was localized with reference to the spinal trigeminal tract and the ventral cochlear nucleus (Paxinos and Watson, 1986). Interpolaris neurons were then viewed under near infrared illumination with a $40 \times$ water-immersion objective (Fluor, $40 \times 10.80 \mathrm{~W}$; Nikon, Mississauga, Ontario, Canada) and a CCD camera (IR-1000; MTI, Michigan City, IN). Large neurons (somata, $>20 \mu \mathrm{m}$ in diameter) were selected for recording. Experiments were conducted at $30-32^{\circ} \mathrm{C}$. Electrodes were pulled from thick wall borosilicate glass $(1.5 / 0.84 \mathrm{~mm}$; WPI, Sarasota, FL) on a horizontal puller (P-97; Sutter Instruments, Novato, CA). The pipette solution contained the following (in mM): 100 K methylsulfate, $15 \mathrm{KCl}, 4 \mathrm{ATP}-\mathrm{Mg}, 0.3 \mathrm{GTP}-\mathrm{Na}_{2}, 10$ creatine phosphate, 0.5 EGTA, 20 HEPES, pH 7.4 with $\mathrm{KOH}, 280 \pm 3 \mathrm{mOsm}$ with sucrose. Electrodes had resistances between 3 and $6 \mathrm{M} \Omega$, and liquid junction potential was not corrected. Whole-cell recordings (seal resistance, $>5$ $\mathrm{G} \Omega$ ) were made at the soma with a Multiclamp 700A amplifier (Molecular Devices, Union City, CA). The series resistance, usually between 10 and $45 \mathrm{M} \Omega$, was compensated using the bridge balance. To build firing rate curves, current steps of $3 \mathrm{~s}$ duration were applied once every $15 \mathrm{~s}$, with increments of 10 or 20 pA. Carbachol (CCh) was applied through the bath, and all solutions were continuously bubbled with $95 \% \mathrm{O}_{2}$ and $5 \% \mathrm{CO}_{2}$. The spinal trigeminal tract was stimulated using a concentric electrode (50 $\mu \mathrm{m}$ tip diameter; FHC, Bowdoinham, ME). Experiments were conducted using the Axograph 4.9 program (Molecular Devices, Palo Alto, CA). Data were filtered at 2 or $4 \mathrm{kHz}$, and digitized at 8 or 16 $\mathrm{kHz}$.

The AxoGraph 4.9 and Origin 7 software (OriginLab, Nothampton, MA) were used for analysis. Action potentials were detected using the event detection package of the AxoGraph. Events with peak amplitude of $60 \mathrm{mV}$ or higher, and a rise time of $\sim 0.5 \mathrm{~ms}$, were detected automatically, and the results were analyzed with Origin 7 . The steady-state spike rate was estimated by counting the number of spikes during the last $2.5 \mathrm{~s}$ of the $3 \mathrm{~s}$ step, and the result was plotted versus the intensity of the injected current ( $F-I$ curve). The slope of the $F-I$ curve (referred to as gain) was estimated by linear fit. Input resistance was measured by applying small current pulses.

Neurons were labeled with biocytin $(0.25 \%)$ included in the pipette solution, and slices were processed for biocytin histochemistry as described previously (Zhang, 2004). Neurons were viewed with a $40 \times$ objective and reconstructed using the Neurolucida system (MicroBrightField, Colchester, VT).

Electrophysiology in vivo. The effect of cholinergic antagonists on the receptive field size of SpVi and VPM neurons was examined in eight rats. Rats were anesthetized with pentobarbital ( $50 \mathrm{mg} / \mathrm{kg})$, supplemented as needed by a small amount of xylazine $(1 \mathrm{mg} / \mathrm{kg})$. The left facial nerve was cut, and the rat was placed in a stereotaxic apparatus. Two stainless-steel tubes (diameter, $1.5 \mathrm{~mm}$; length, $15 \mathrm{~mm}$; spacing, $10 \mathrm{~mm}$ ) were fixed across the surface of the skull by means of screws and acrylic cement. For the recording session, the rat's head was maintained in a stereotaxic position by means of a small U-shaped frame bearing adjustable pins inserted in the tube openings of the cemented device. The frame was secured to a large steel post so that whiskers on the left mystacial pad were freely accessible for stimulation. Before the start of recordings, the nape of the neck was infiltrated with a long-lasting local anesthetics (Marcaine $1 \%$ ) to reduce animal discomfort. During the recording sessions, animals frequently displayed spontaneous twitches of the right whiskers, briskly reacted to a moderate pinch of the hindlimb, but otherwise remained motionless, indicating that they did not experience any discomfort. An 
additional dose of anesthetics (ketamine, $20 \mathrm{mg} / \mathrm{kg}$ ) was given when small amplitude whisking movements were noticed.

Single units were recorded with glass micropipettes $(0.5-1 \mu \mathrm{m})$ filled with potassium acetate $(0.5 \mathrm{M})$. Signals were amplified, bandpass filtered $(100 \mathrm{~Hz}$ to $3 \mathrm{kHz}$ ), and sampled at $10 \mathrm{kHz}$. The receptive field size of single units was estimated by deflecting individual whiskers with a handheld probe under a dissecting microscope. Whiskers were cut at $2 \mathrm{~cm}$ from the skin, and care was taken not to stimulate intervibrissa fur or the mystacial pad itself. Once a representative sample of units was obtained, scopolamine (a muscarinic antagonist, $5 \mathrm{mg} / \mathrm{kg}$ ) or mecamylamine (a nicotinic antagonist, $5 \mathrm{mg} / \mathrm{kg}$ ) were administered intraperitoneally, and receptive field sizes were assessed over the next $2 \mathrm{~h}$.

In additional experiments $(n=6)$, we also examined the effect of local application of CCh on whisker-evoked responses in the SpVi. Rats were prepared as described above, and a midline brainstem lesion was performed before the recording session to reduce the receptive field size of SpVi neurons. The lesioning procedure was identical with that described by Timofeeva et al. (2004). After the lesion, most interpolaris cells responded to a single vibrissa, which was further ascertained by means of air jet stimuli to simultaneously deflect a large number of vibrissas. First, a peristimulus time histogram (PSTH) was built by compiling 20 responses (bin width, $1 \mathrm{~ms}$ ) to air-jet stimuli. Then, the effective whisker was inserted into a $2 \mathrm{~mm}$ glass capillary, and a second PSTH was built. The capillary gently pressed against the pad to completely mask the whisker. Air jets were generated by a Picospitzer (General Valve, Brookshire, TX) connected to a pipette (tip diameter, $\sim 500 \mu \mathrm{m}$ ). The pipette was positioned at a distance of $4-5 \mathrm{~cm}$ of the vibrissas, in front of the animal, and the air jet was directed away from the rat's face to avoid as much as possible stimulating other orofacial afferents. This created a cone-shaped air displacement that deflected six to seven whiskers about the effective whisker. The delay between the command voltage and the actual motion of vibrissas was measured by placing a piezoelectric film (Measurement Specialties, Fairfield, NJ) at the same distance from the tip of the micropipette. This delay was subtracted from the recordings to build PSTHs of sensory-evoked responses.

Cells were recorded with micropipettes (tip diameter, $1.5 \mu \mathrm{m}$ ) filled with $100 \mathrm{~mm}$ CCh in $0.9 \% \mathrm{NaCl}$. Receptive field sizes of well isolated single units were assessed before and after pressure application of CCh in the vicinity of the cells. To prevent loosing cells during drug ejection, pressure was increased gradually (up to $20 \mathrm{psi}$; Picospritzer) until a sudden change in spike amplitude indicates the actual outflow of the solution. As estimated under microscopic control, the amount of solution ejected ranged from 10 to $20 \mathrm{nl}$.

Data analysis was performed with the NeuroExplorer (Plexon, Dallas, TX) and Excel (Microsoft, Redmond, WA) software. Throughout this article, results are reported as means $\pm \mathrm{SD}$, and statistical analysis was done using Student's $t$ test.

\section{Results}

\section{Cholinergic innervation of the SpVi}

To identify the putative modulatory system(s) that controls the receptive field size of interpolaris neurons, FG was injected in the ventral, whisker-responsive region of the SpVi (Fig. 1A,B). As reported in previous studies, a large number of retrogradely labeled cells were found within the trigeminal column itself (mostly in the caudalis and oralis divisions) and in the parabrachial nucleus (Hallas and Jacquin, 1990; Jacquin et al., 1990a; Yoshida et al., 1997; Dallel et al., 2004). Some retrogradely labeled cells were also scattered in the gigantocellular reticular nucleus and raphe magnus, but the most conspicuous population of labeled neurons (100-150 cells per rat) was found bilaterally in the pedunculopontine tegmental region (PPTg) (Fig. 1C,D).

Because PPTg is known as the primary source of cholinergic input to the thalamus and brainstem (Sofroniew et al., 1985; Woolf and Butcher, 1986, 1989), we examined whether retrogradely labeled cells were cholinergic. Dual immunostaining for FG and ChAT confirmed that indeed a large proportion ( 70\%)
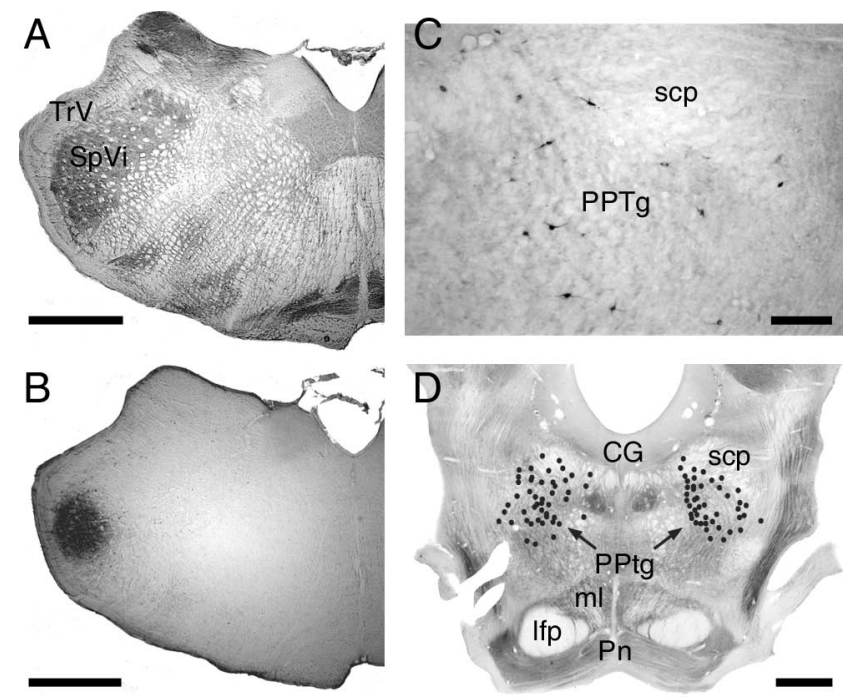

Figure 1. Retrograde labeling of PPTg neurons after FG injection in the SpVi. $A, B$, Alternate sections at the injection site were stained for cytochrome oxidase $(\boldsymbol{A})$ and FG immunoreactivity (B). C, Photomicrograph of FG-labeled cells in the PPTg. D, Bilateral distribution of retrogradely labeled cells in the mesopontine area; each cell labeled within a tissue slab $\sim 500 \mu \mathrm{m}$ thick is represented by a dot. Note that only one-half of the sections (70 $\mu \mathrm{m}$ thick) were immunostained for FG. Scale bars: $\boldsymbol{A}, \boldsymbol{B}, \boldsymbol{D}, 1 \mathrm{~mm} ; \boldsymbol{C}, 100 \mu \mathrm{m}$. CG, Central gray; Ifp, Iongitudinal fasciculus of the pons; ml, medial lemniscus; $\mathrm{Pn}$, pontine nuclei; scp, superior cerebellar peduncle; $\mathrm{TrV}$, spinal trigeminal tract.

of SpVi-projecting PPTg neurons were also ChATimmunoreactive (Fig. 2A,B). Direct evidence for a cholinergic innervation of the SpVi was further provided by VAChT immunostaining, which revealed the presence of numerous immunoreactive terminals in the $\mathrm{SpVi}$ and also, although to a lesser degree, in the other trigeminal nuclei. Figure $2 C-E$ shows the distribution of VAChT-positive terminals among SpVi neurons retrogradely labeled from the PrV; one can note that immunostained terminals often form strings or clusters (Fig. 2E, arrows) in close vicinity of some of the labeled somata.

To determine whether acetylcholine is released at synapses or in a paracrine manner, VAChT-immunostained terminals were examined at the electron microscopic level. Among the 37 terminals studied, 36 established symmetric $(n=24)$ or asymmetric $(n=12)$ synaptic contacts, and 1 terminal that was examined in six serial ultrathin sections had no postsynaptic target. Symmetric contacts involved dendritic appendages $(n=4)$, dendritic shafts $(n=14)$, and axon terminals containing round vesicles $(n=6)$. Asymmetric contacts were observed on dendritic appendages $(n=9)$ and dendritic shafts $(n=3)$. Figure 3 provides representative examples of the above-mentioned types of synapses. Synaptic profiles shown in Figure $3 E$ are particularly interesting, in that a VAChT-positive terminal takes part in a triadic arrangement, where it contacts a dendrite and an axon terminal, which is itself presynaptic to the same dendrite. In sum, ultrastructural data show that cholinergic axons make classic excitatory and inhibitory synaptic contacts on interpolaris cells, and further indicate that cholinergic afferents also act presynaptically.

\section{CCh excites large-sized interpolaris cells in slices}

Previous studies showed that the SpVi contains two broad classes of cells: medium- to large-sized neurons that project to the thalamus, tectum, or cerebellum (e.g., projection cells), and smallsized local circuit cells with extensive intersubnuclear projections (Jacquin et al., 1989a,b). We selected the former neurons in slices 
(soma, $>20 \mu \mathrm{m}$ in diameter) to examine with whole-cell patch-clamp recordings the modulatory action of CCh. These neurons $(n=13)$ had a mean resting potential of $-60.8 \pm 1.6 \mathrm{mV}$, and a mean input resistance of $39 \pm 12 \mathrm{M} \Omega$. Depolarizing current steps induced regular firing with little accommodation, and all neurons displayed a prominent sag in response to large hyperpolarizing current steps, presumably mediated by the activation of a hyperpolarization-activated cation current (Fig. 4A). Eleven neurons were stained with biocytin, and reconstructions revealed cells with rostrocaudally oriented dendritic fields that extended mediolaterally over an area much wider than the average diameter of a single barrelette (e.g., $\sim 60 \mu \mathrm{m}$ ) (Fig. $4 \mathrm{~B}$ ); thus, these neurons likely belong to the class of projection cells (Jacquin et al., 1986, 1989a,b).

Bath applications of CCh $(20 \mu \mathrm{M})$ strongly depolarized all neurons tested $(n=10)$ and induced tonic discharges in seven neurons (Fig. 4C). This effect was reversible and was associated with a significant increase in input resistance (88 \pm $24 \%>$ predrug value); $n=6$ cells; $p<$ 0.01 , paired $t$ test). To further examine the effects of CCh on neuronal excitability, depolarizing current steps were applied before, during, and after CCh application, while the baseline membrane potential was maintained at $-62 \mathrm{mV}$ throughout the recording with DC current injection. Carbachol substantially reduced the current required to trigger trains of action potentials (Fig. 4D). As shown by the firing rate curves ( $F-I$ curves) (Fig. $4 E$ ), CCh reversibly shifted the $F-I$ curves to the left but had little effect on their slope ( $n=6$ cells; $p>0.1$, paired $t$ test). We also examined whether $\mathrm{CCh}$ enhanced synaptic transmission at the trigeminal ganglion-SpVi synapse. Stimulation of the spinal trigeminal tract evoked large amplitude unitary EPSCs with fast decay times at a holding potential of $-60 \mathrm{mV}$ (Fig. $4 \mathrm{~F}$ ), and CCh had little effect on the peak amplitude of evoked EPSCs $(91 \pm 6 \%$ of the control; $n=4$ cells). Thus, these results indicate that CCh acts mainly postsynaptically on large-sized interpolaris cells by a mechanism that is reminiscent of the muscarinic action observed in other regions of the brain (McCormick and Prince, 1987; McCormick et al., 1993).

\section{Cholinergic modulation of receptive field size}

In light of the above data, one would expect local application of CCh in vivo to increase cell excitability and enlarge receptive field size in the SpVi. We thus applied CCh on interpolaris neurons that had been rendered monowhisker responsive by a midline brainstem lesion. Figure $5 A$ shows a representative unit that was driven by air jet deflection of whisker D2. After inserting the effective whisker into a glass capillary, responses were obliterated (Fig. $5 B$ ), confirming that this unit solely responded to whisker D2. Then, CCh was ejected from the recording pipette (60 s pulse; 10 psi) while monitoring whisker-evoked responses with whisker D2 still masked. Within 2 min after the onset of drug application, the frequency of resting discharges slightly increased and adjacent whisker deflection started eliciting responses (Fig. 5C). Over the next minute, the rate of background discharges further increased, and after demasking whisker D2, responses were markedly enhanced (Fig. 5D). The effect of CCh lasted for $>15 \mathrm{~min}$ and could be abbreviated by systemic administration of scopolamine, which reduced the receptive field back to whisker D2 alone (Fig. 5E,F). Three other cells were tested in this manner with similar results. Population PSTHs in Figure 5G-I summarize the effect of CCh on 14 interpolaris cells that had been rendered monowhisker responsive by a midline lesion. Air jet stimulation produced robust responses (Fig. $5 G$ ) that were abolished by masking the effective whisker (Fig. 5H). Within 2-5 min after CCh application, while the effective whisker was still masked, cells started responding to adjacent whiskers (Fig. $5 I)$.

In additional experiments ( $n=3$ rats), the receptive field size of interpolaris cells was assessed before and after systemic administration of scopolamine in normal rats, without brainstem lesion. During the predrug period, cells responded, on average, to $5.96 \pm 2.88$ whiskers, but within the first hour after scopolamine injection, receptive field sizes were significantly reduced to $1.40 \pm 0.77$ whisker $(p<0.05$, unpaired $t$ test $)$. The depressive effect started $\sim 10$ min after drug administration, was maximal 20 min later, and partly recovered over the next $2 \mathrm{~h}$ (Fig. $6 \mathrm{~A}$ ). In the course of these experiments, the receptive field of two units was repeatedly assessed while the muscarinic block progressed; before scopolamine injection, these units responded to 11 and 6 whiskers, respectively, but 25 min after drug injection, receptive fields were reduced to 3 whiskers (data points connected by lines in Fig. $5 A$ ). In contrast, the administration of a nicotinic antagonist, 


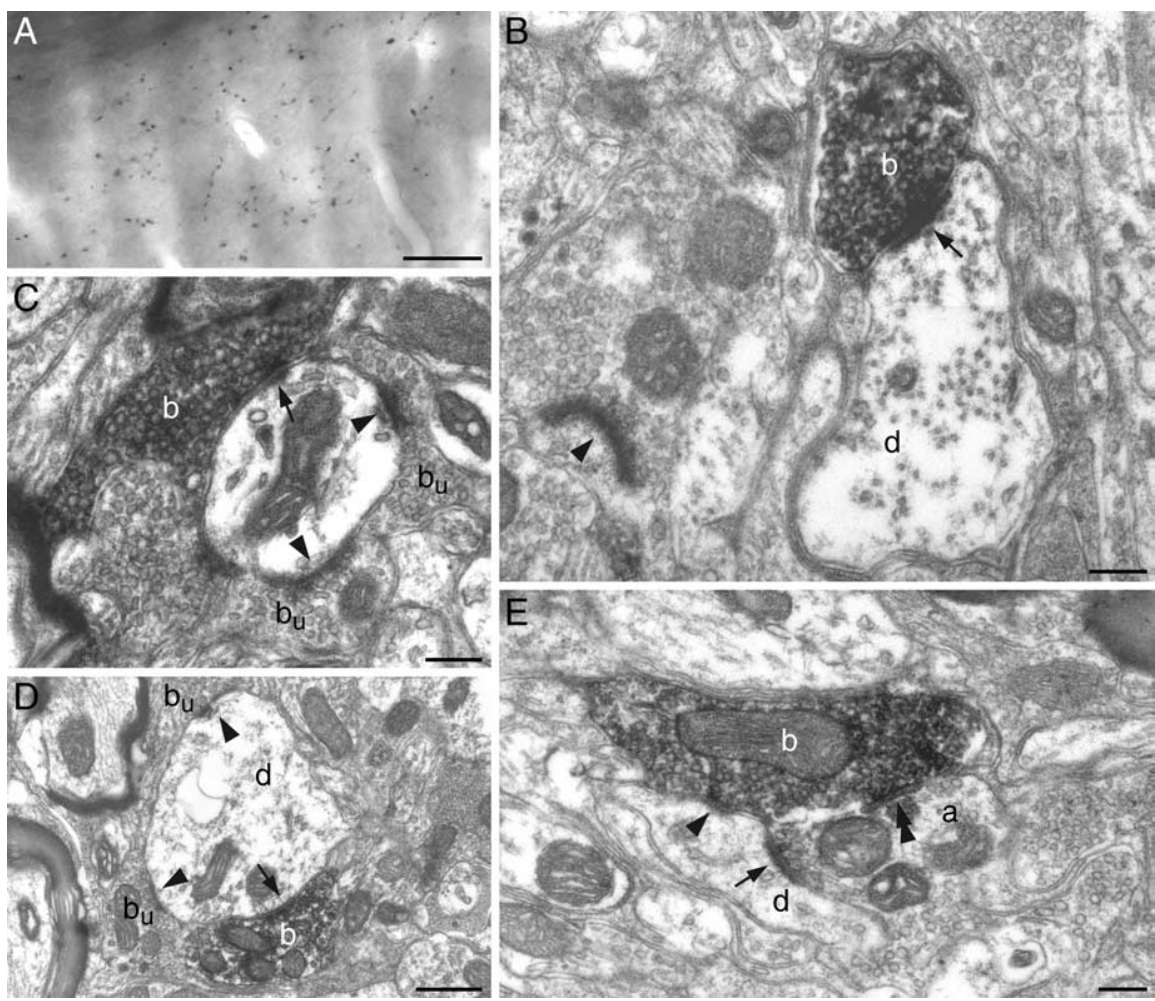

Figure 3. Ultrastructure of VAChT-immunostained terminals in the SpVi. $\boldsymbol{A}$, Light photomicrograph of VAChT-positive terminals in the SpVi. $\boldsymbol{B}$, VAChT-immunoreactive bouton (b) forming an asymmetric synapse (arrow) on a small caliber dendrite (d). $\boldsymbol{C}$, Asymmetric synapses made by a VAChT-immunopositive bouton ( $b$; arrow) and an immunonegative bouton ( $b_{u}$; arrowheads) on a dendrite. $\boldsymbol{D}$, Symmetric axodendritic synapses between VAChT-positive $(b)$ and VAChT-negative $\left(b_{u}\right)$ boutons at sites marked by an arrow and arrowheads, respectively. $\boldsymbol{E}$, An unlabeled axon terminal (a) forms an asymmetric synapse on a small caliber dendrite (d; arrow). The same dendrite receives a symmetric contact (arrowhead) from a VAChT-immmunopositive bouton (b), which also forms a symmetric synapse with the unlabeled axon terminal (double arrowhead). Scale bars: $A, 20 \mu \mathrm{m} ; \boldsymbol{B}, \boldsymbol{C}, \boldsymbol{E}, 0.2 \mu \mathrm{m} ; \boldsymbol{D}, 0.5 \mu \mathrm{m}$. ceptive field size in the SpVi. It appeared unlikely that axotomy of ascending interpolaris axons might have caused such an effect, because a similar shrinkage of receptive fields was observed after midline lesions made rostrally to the crossing of SpVi axons, at the level of the oralis nucleus (our unpublished data). The severing of corticofugal axons, which travel medially in the brainstem, or lesion of serotonergic afferents to the SpVi neither appeared as plausible explanations because barrel cortex lesion or serotonine depletion do not reduce receptive field size in the SpVi (Jacquin et al., 1990b; Misra and Klein, 1995). Thus, in light of the present results, it seems most likely that the depressive effect of a midline lesion results from the severing of cholinergic axons or from a marked diminution of their activity.

Tract tracing and single-cell labeling studies have shown that the SpVi contains two broad classes of neurons: medium- to large-sized neurons that project to the thalamus or to the cerebellum, and smallsized local circuit cells that project to the other divisions of the trigeminal complex (Jacquin et al., 1989a,b, 1990a). Projection cells have dendritic fields encompassing multiple barrelettes, and they respond to the deflection of several whiskers. In contrast, local circuit cells have narrow dendritic fields, about the size of a barrelette, and under moderate to deep barbiturate mecamylamine (two rats), did not produce any significant change in receptive field size $(4.78 \pm 2.26$ and $5.75 \pm 2.49$ whiskers, before and after mecamylamine, respectively) (Fig. 6B).

Because adjacent whisker responses in the lemniscal pathway relies on projections from the spinal complex to the PrV (Timofeeva et al., 2004), we tested whether scopolamine also reduced receptive field size in the VPM. Within $1 \mathrm{~h}$ after scopolamine administration, the average size of receptive fields in the VPM passed from $3.57 \pm 1.36$ to $1.26 \pm 0.58$ whiskers, and it recovered to an average value of $2.86 \pm 1.73$ whiskers $2 \mathrm{~h}$ after drug injection (Fig. 6C). Again, the suppressive effect was statistically significant $(p<0.05$, unpaired $t$ test) for the first hour after the injection.

\section{Discussion}

The present study provides tract tracing, immunocytochemical, and ultrastructural evidence for a cholinergic innervation of the SpVi by PPTg neurons. Recordings in slices showed that CCh depolarizes large-sized interpolaris cells and increases their excitability. Local application of CCh in vivo enhances responses to adjacent whiskers, whereas systemic administration of scopolamine produces an opposite effect. Together, these results show that mesopontine cholinergic neurons exert a direct, effective control over receptive field size at the very first relay stations of the vibrissal system in rodents.

\section{Synthesis of receptive fields in the $\mathrm{SpVi}$}

The starting point of the present study was the observation that a midline brainstem lesion produced a dramatic reduction of re- anesthesia, they respond to a single whisker. Although the structure of receptive fields seems related to the way dendritic arbors cut across the array of barrelettes, it should be pointed out that just like primary afferent fibers, intersubnuclear axons also form barrelette-like terminal fields in the SpVi (Jacquin et al., 1990a; Timofeeva et al., 2004). Thus, both groups of axons can potentially contribute to receptive field synthesis. If the structure of receptive fields merely relied on the convergence of peripheral afferents, one could hardly explain why, under scopolamine, a given whisker remains effective when combined deflection of several whiskers does not produce postsynaptic depolarization large enough to trigger cell discharges. It might well be that adjacent whisker responses are mediated, at least in part, by local or intersubnuclear connections whose synaptic efficacy depends on a muscarinic modulation.

That ACh might modulate sensory responses in the SpVi was not completely unexpected, because autoradiographic studies have reported previously that this nucleus contains a fair density of muscarinic and nicotinic binding sites (Wamsley et al., 1981; Rotter, 1984; Cortes and Palacios, 1986; Vilaro et al., 1992). The strong impact of this modulation at a very first relay station of a sensory system was unexpected, however, as was the diversity of synaptic contacts revealed by electron microscopy. Actually, cholinergic terminals were seen to establish asymmetric contacts with dendrites, and symmetric contacts with dendrites and axon terminals, suggesting a diversity of cell-specific excitatory and inhibitory actions. In the present study, none of the postsynaptic targets has been identified at the electron microscopic level, but there is little 
doubt that cholinergic terminals do contact projection cells, because these large-sized neurons were consistently depolarized by $\mathrm{CCh}$ in slices. This contention is also supported by the fact that scopolamine administration in vivo produces a marked shrinkage of the receptive field of the interpolaris cells that normally respond to several whiskers. Other axodendritic contacts might involve local circuit cells, but the origin of the terminals contacted by cholinergic axons remains elusive. Indeed, CCh application in slice little depressed synaptic currents generated by the activation of primary afferent fibers. Thus, the presynaptic action of ACh likely targets terminals of central origin, some of which might not necessarily be involved in vibrissal information processing (parabrachial inputs, for example).

\section{Methodological considerations}

An exhaustive survey of the effect of CCh on interpolaris cells in slices was beyond the scope of the present study, which focused on projection cells. It is very likely that a number of these cells with extensive dendritic arbors did not survive in slices, which is indicated by the fact that only two to three "healthy" large neurons were present in each slice. However, the effect of $\mathrm{CCh}$ on these cells was very consistent, whereas cells with small-sized somata exhibited a broader spectrum of responses that are currently under investigation.

The receptive field size of $\mathrm{SpVi}$ and VPM neurons was assessed with a handheld probe. This approach permits a rapid scan of receptive fields by deflecting a number of single vibrissa in different directions, but it may yield to an underestimate of receptive field size, particularly when spontaneous activity is high and responses weak. However, in our recording conditions (facial nerve cut), spontaneous activities were low, and receptive field sizes of SpVi and VPM neurons before scopolamine administration were quantitatively similar to those estimated in previous studies by means of controlled whisker deflection (Woolston et al., 1982; Friedberg et al., 1999; Minnery et al., 2003; Timofeeva et al., 2004). Moreover, the reduction of receptive fields to a single vibrissa after scopolamine was also assessed in a subset of neurons with air jet stimuli. Therefore, the lack of responses when the effective whisker was masked strongly supports results obtained after manual whisker deflection.

\section{Functional implications}

Pedunculopontine projections to the SpVi introduce an additional complexity when it comes to interpret the effects of highfrequency stimulation of this cholinergic group on sensory transmission in the thalamus and cortex. Activation of the PPTg may indeed change synaptic efficacy in trigeminal nuclei, which calls
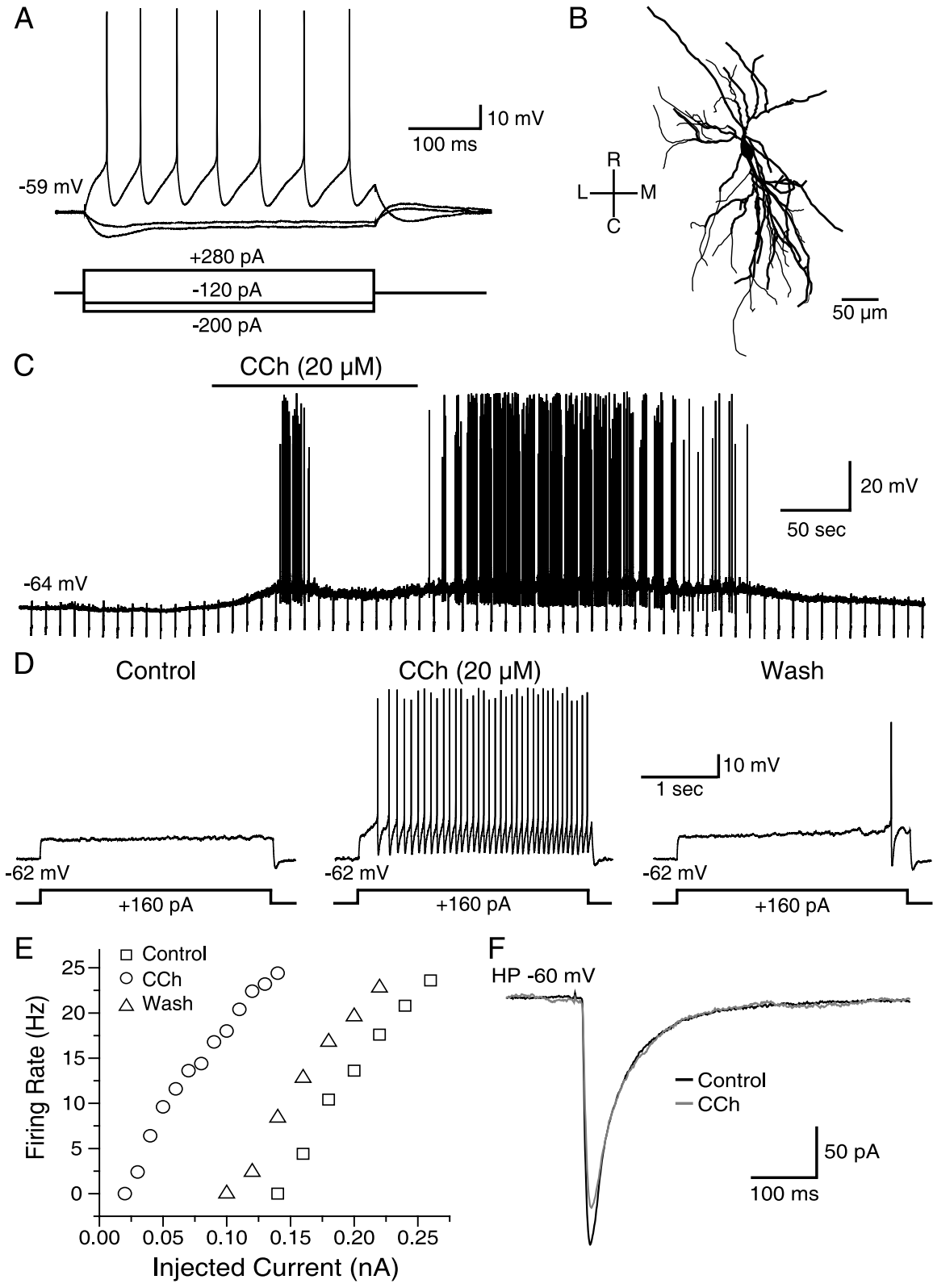

F

Figure 4. Intrinsic properties and responses of large-sized interpolaris cells to CCh application in slices. $\boldsymbol{A}$, Cellular responses to depolarizing and hyperpolarizing current steps; note the prominent sag during membrane hyperpolarization. $\boldsymbol{B}$, Morphology of the same neuron revealed by the intracellular injection of biocytin. C, Caudal; L, lateral; M, medial; R, rostral. C, Bath application of CCh (20 $\mu \mathrm{m})$ depolarizes large-sized SpVi neurons and induces action potentials; note the marked increase in input resistance associated with the depolarization. D, Responses to depolarizing current steps before, during, and 10 min after CCh application (20 $\mu \mathrm{m})$. The baseline level was maintained at $-62 \mathrm{mV}$ throughout the recording. $\boldsymbol{E}, F-/$ curves obtained from another neuron before, during, and $10 \mathrm{~min}$ after CCh application $(20 \mu \mathrm{M})$. Here again, the resting membrane potential was maintained at $-60 \mathrm{mV}$ throughout the test. Carbachol reversibly shifts the $F-I$ curve to the left with little effect on the slope. $F$, Monosynaptic EPSCS evoked by stimulating the spinal trigeminal tract before (black trace) and during (Ch application (gray trace).

for adequate control to ascertain the locus of cholinergic modulation. This qualification also applies to our results obtained in the VPM after scopolamine administration. Although the reduction of receptive fields in the thalamus is in line with that observed in the brainstem, response suppression in the VPM may also be ascribed, at least in part, to a blockade of the muscarinic modulation in the thalamus itself.

Deep anesthesia has long been suspected as a factor that strongly depresses adjacent whisker responses in brainstem and thalamus (Waite, 1973; Simons and Carvell, 1989; ArmstrongJames and Callahan, 1991; Friedberg et al., 1999), but the reason 

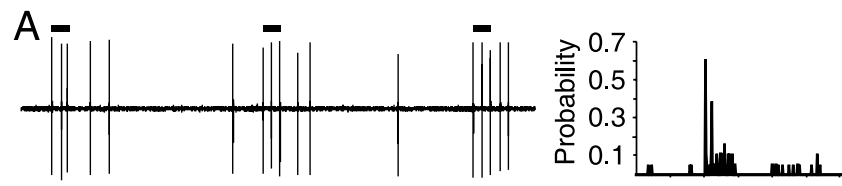

B
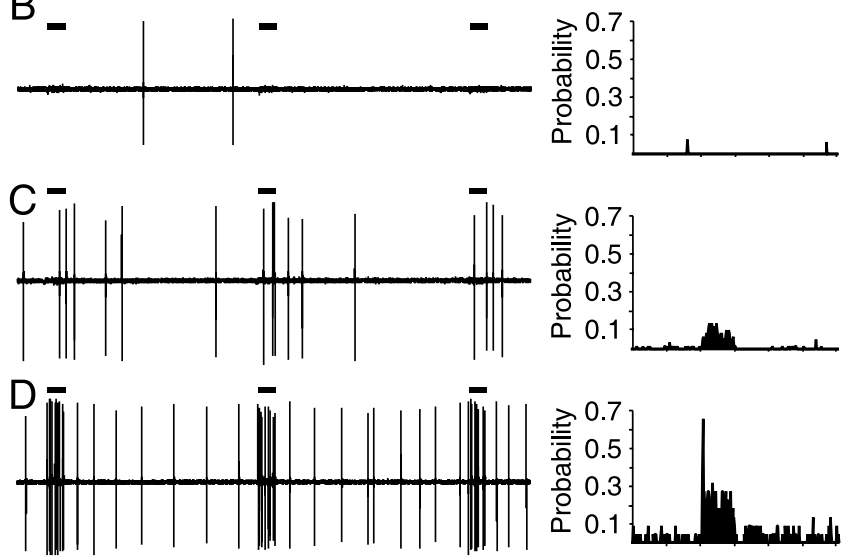

E
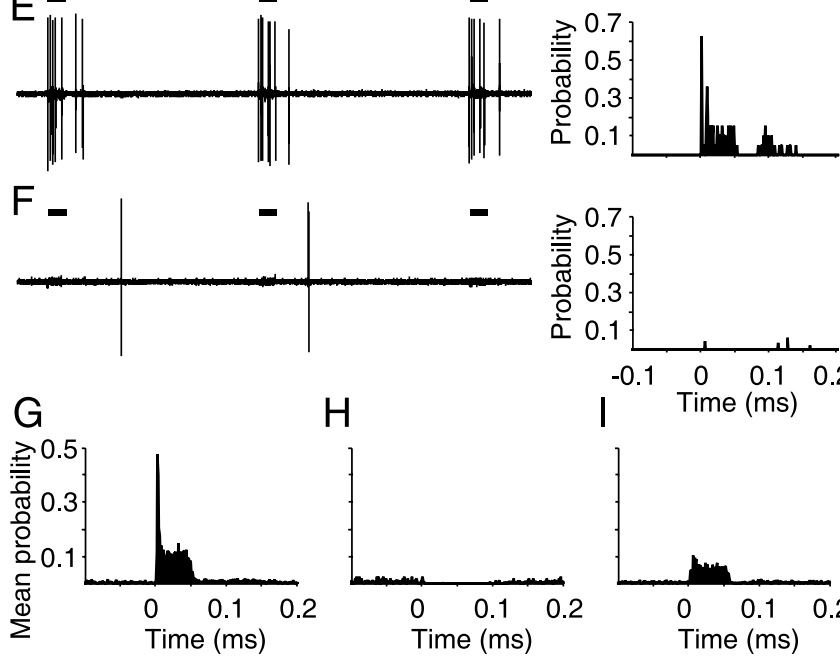

Figure 5. Effect of local application of CCh on whisker-evoked responses in the SpVi. Traces $\boldsymbol{A}-\boldsymbol{F}$ and associated right-hand PSTHs show responses of a D2-responsive unit recorded in a rat after a brainstem midline lesion. $\boldsymbol{A}$, Control responses to an air jet (black bars). $\boldsymbol{B}$, Lack of responses after inserting whisker D2 within a glass capillary. $C$, Responses evoked 3 min after local application of CCh with whisker D2 still masked and then demasked (D). E, Responses recorded 5 min after systemic administration of scopolamine with all whiskers free to move and after remasking whisker D2 $(\boldsymbol{F})$. Population PSTHs at the bottom show the responses of 14 monowhisker responsive cells before $(\boldsymbol{G})$ and after $(\boldsymbol{H})$ masking the effective whisker within a glass capillary. The PSTH in I shows adjacent whisker responses (principal whisker still masked) evoked in the same cells after local application of CCh.

for which depression already occurs at the first relay station of the lemniscal pathway (in the $\operatorname{PrV}$ ) remained obscure. The disynaptic or polysynaptic circuits that mediate these responses may explain, at least in part, their vulnerability to anesthetics, but the well known depressive action of most anesthetics (particularly barbiturates) on cholinergic transmission likely represents the principal factor (Smaje, 1976; Pape and Eysel, 1988; Puil and el-Beheiry, 1990). In this respect, it is noteworthy that local circuit interpolaris cells respond to a single vibrissa under barbiturate anesthesia (Jacquin et al., 1989a), but that very few monowhisker responsive cells were found in rats maintained under light ketamine anesthesia (Timofeeva et al., 2004; the present study). Whether this discrepancy depends on a sampling bias or
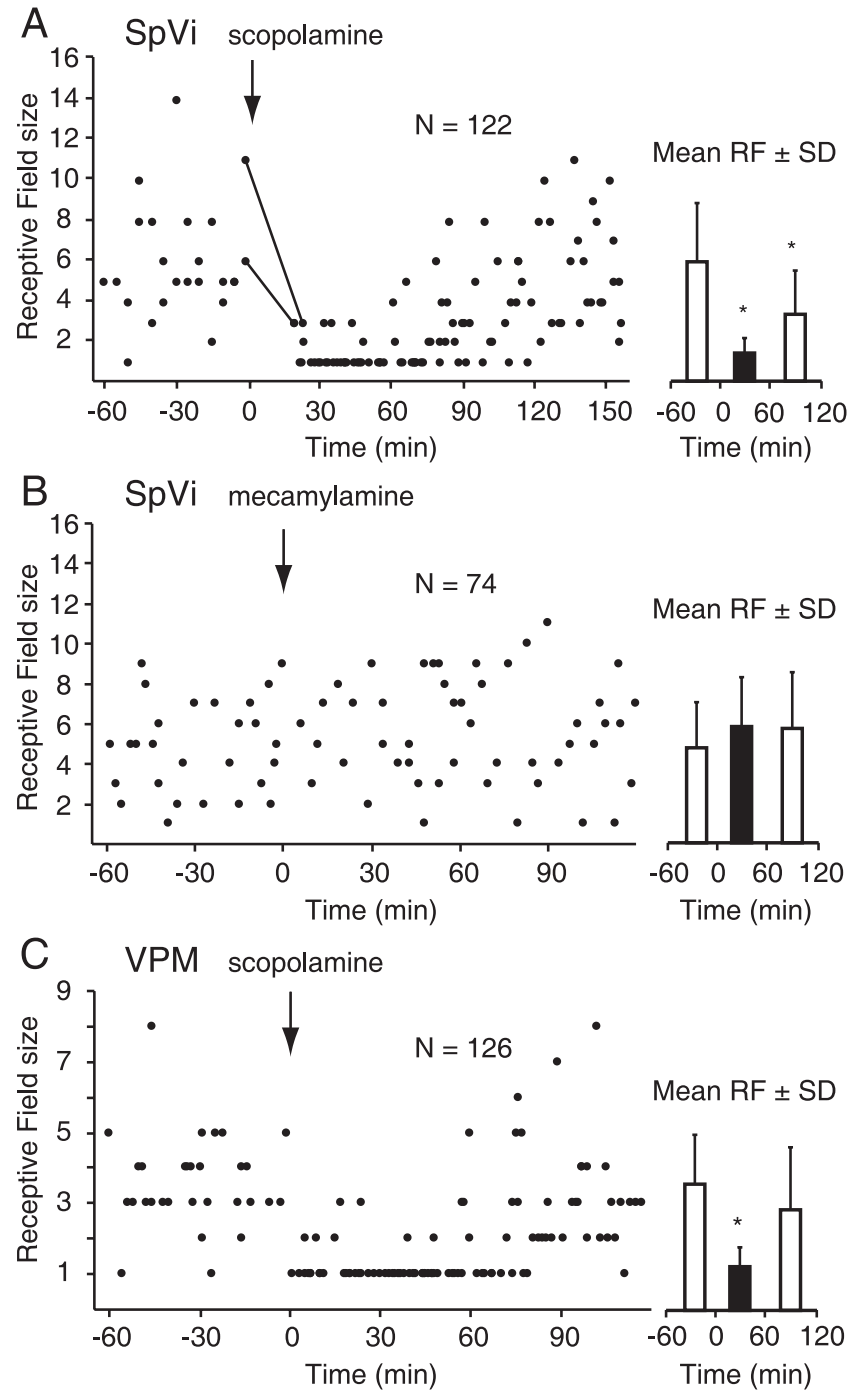

Figure 6. Effect of systemic administration of cholinergic antagonists on the receptive field size of SpVi and VPM neurons. The receptive field size of SpVi neurons was reduced by scopolamine administration $(\boldsymbol{A})$ but not by mecamylamine $(\boldsymbol{B})$. Scopolamine also reduced the receptive field size of VPM neurons $(\boldsymbol{C})$. Each dot in the scatter plots represents a unit; the scatter plot in $\boldsymbol{A}$ combines the results of three experiments, and each of the scatter plots in $\boldsymbol{B}$ and $\boldsymbol{C}$ combines the results of two experiments. Data points connected by lines in $\boldsymbol{A}$ represent the same units. Drugs were injected intraperitoneally at time 0 . Right-hand histograms show average receptive field sizes computed before drug injection and during the first and second hours after drug administration (the asterisk indicates a significant difference between predrug and postdrug periods with $p<0.05$, unpaired $t$ test). Error bars indicate SD. RF, Receptive field.

on the type of anesthetics used remains an important issue to settle.

In sensory systems, it is usually considered that cholinergic projections from the PPTg exert their modulatory action at thalamic level, where they enhance the relay of sensory messages during arousal (Kayama et al., 1986; McCormick and Prince, 1987; Hu et al., 1989; Deschênes and Hu, 1990; Curro Dossi et al., 1991; McCormick, 1992; Oldford and Castro-Alamancos, 2003; Mooney et al., 2004). In prethalamic stations, sensory transmission is traditionally thought not to be state-dependent, but in light of the present results, that view may now require revision. Collectively, PPTg cells are most active during states of electroencephalographic desynchronization, and there exists a number of findings linking ACh release from the PPTg to the electrophysiological and behavioral manifestations of REM sleep (for review, 
see McCarley, 2004). For the moment, we have very little insight into the role(s) of the SpVi in the vibrissal system, but there is metabolic evidence that this nucleus gets activated during selfgenerated whisker motion (Sharp and Evans, 1982; Jacquin et al., 1993). Considering the behavioral importance of information gathered through whisker motion in rodents (Welker, 1964), it is perhaps not surprising that subtle shifts in arousal level produce immediate vibrissa reactions and rapid excitability adjustments at the very first relay stations of this system. A close relationship between circuits involved in arousal and those involved in early processing of vibrissal information is further suggested by the occurrence of vibrissa twitches together with rapid eye movements during paradoxical sleep in rodents (Vanderwolf, 1969; Lerma and Garcia-Austt, 1985).

\section{References}

Armstrong-James M, Callahan CA (1991) Thalamo-cortical processing of vibrissal information in the rat. II. Spatiotemporal convergence in the thalamic ventroposterior medial nucleus (VPm) and its relevance to generation of receptive fields of S1 cortical "barrel" neurones. J Comp Neurol 303:211-224.

Cortes R, Palacios JM (1986) Muscarinic cholinergic receptor subtypes in the rat brain. I. Quantitative autoradiographic studies. Brain Res 362:227-238.

Curro Dossi R, Paré D, Steriade M (1991) Short-lasting nicotinic and longlasting muscarinic depolarizing responses of thalamocortical neurons to stimulation of mesopontine cholinergic nuclei. J Neurophysiol 65:393-406.

Dallel R, Ricard O, Raboisson P (2004) Organization of parabrachial projections from the spinal trigeminal nucleus oralis: an anterograde tracing study in the rat. J Comp Neurol 470:181-191.

Deschênes M, Hu B (1990) Membrane resistance increase induced in thalamic neurons by stimulation of brainstem cholinergic afferents. Brain Res 513:339-342.

Friedberg MH, Lee SM, Ebner FF (1999) Modulation of receptive field properties of thalamic somatosensory neurons by the depth of anesthesia. J Neurophysiol 81:2243-2252.

Hallas BH, Jacquin MF (1990) Structure-function relationships in rat brain stem subnucleus interpolaris. IX. Inputs from subnucleus caudalis. J Neurophysiol 64:28-45.

Henderson TA, Jacquin MF (1995) What makes subcortical barrels? In: Cerebral cortex, Vol 12, The barrel cortex of rodents (Jones EG, Diamond IT, eds), pp 123-187. New York: Plenum.

Hu B, Steriade M, Deschênes M (1989) The effects of brainstem peribrachial stimulation on neurons of the lateral geniculate nucleus. Neuroscience 31:13-24.

Jacquin MF, Mooney RD, Rhoades RW (1986) Morphology, response properties, and collateral projections of trigeminothalamic neurons in brainstem subnucleus interpolaris of rat. Exp Brain Res 61:457-468.

Jacquin MF, Golden J, Rhoades RW (1989a) Structure-function relationships in rat brainstem subnucleus interpolaris: III. Local circuit neurons. J Comp Neurol 282:24-44.

Jacquin MF, Barcia M, Rhoades RW (1989b) Structure-function relationships in rat brainstem subnucleus interpolaris: IV. Projection neurons. J Comp Neurol 282:45-62.

Jacquin MF, Chiaia NL, Haring JH, Rhoades RW (1990a) Intersubnuclear connections within the rat trigeminal brainstem complex. Somatosens Mot Res 7:399-420.

Jacquin MF, Wiegand MR, Renehan WE (1990b) Structure-function relationships in rat brain stem subnucleus interpolaris. VIII. Cortical inputs. J Neurophysiol 64:3-27.

Jacquin MF, McCasland JS, Henderson TA, Rhoades RW, Woolsey TA (1993) 2-DG uptake patterns related to single vibrissae during exploratory behaviors in the hamster trigeminal system. J Comp Neurol 332:38-58.

Kayama Y, Takagi M, Ogawa T (1986) Cholinergic influence of the laterodorsal tegmental nucleus on neuronal activity in the rat lateral geniculate nucleus. J Neurophysiol 56:1297-1309.
Kwegyir-Afful EE, Bruno RM, Simons DJ, Keller A (2005) The role of thalamic inputs in surround receptive fields of barrel neurons. J Neurosci 25:5926-5934.

Lerma J, Garcia-Austt E (1985) Hippocampal theta rhythm during paradoxical sleep. Effects of afferent stimuli and phase relationships with phasic events. Electroencephalogr Clin Neurophysiol 60:46-54.

McCarley RW (2004) Mechanisms and models of REM sleep control. Arch Ital Biol 142:429-467.

McCormick DA (1992) Cellular mechanisms underlying cholinergic and noradrenergic modulation of neuronal firing mode in the cat and guinea pig dorsal lateral geniculate nucleus. J Neurosci 12:278-289.

McCormick DA, Prince DA (1987) Actions of acetylcholine in the guineapig and cat medial and lateral geniculate nuclei, in vitro. J Physiol (Lond) 392:147-165.

McCormick DA, Wang Z, Huguenard J (1993) Neurotransmitter control of neocortical neuronal activity and excitability. Cereb Cortex 3:387-398.

Minnery BS, Simons DJ (2003) Response properties of whisker-associated trigeminothalamic neurons in rat nucleus principalis. J Neurophysiol $89: 40-56$

Minnery BS, Bruno RM, Simons DJ (2003) Response transformation and receptive field synthesis in the lemniscal trigeminothalamic circuit. J Neurophysiol 90:1556-1570.

Misra BR, Klein BG (1995) Functional properties of cells in rat trigeminal subnucleus interpolaris following local serotonergic deafferentation. Somatosens Mot Res 12:11-28.

Mooney DM, Zhang L, Basile C, Senatorov VV, Ngsee J, Omar A, Hu B (2004) Distinct forms of cholinergic modulation in parallel thalamic sensory pathways. Proc Natl Acad Sci USA 101:320-324.

Oldford E, Castro-Alamancos MA (2003) Input-specific effects of acetylcholine on sensory and intracortical evoked responses in the "barrel cortex" in vivo. Neuroscience 117:769-778.

Pape HC, Eysel UT (1988) Cholinergic excitation and inhibition in the visual thalamus of the cat: influences of cortical inactivation and barbiturate anesthesia. Brain Res 440:79-86.

Paxinos G, Watson C (1986) The rat brain in stereotaxic coordinates. San Diego: Academic.

Puil E, el-Beheiry H (1990) Anaesthetic suppression of transmitter actions in neocortex. Br J Pharmacol 101:61-66.

Rhoades RW, Belford GR, Killackey HP (1987) Receptive-field properties of rat ventral posterior medial neurons before and after selective kainic acid lesions of the trigeminal brain stem complex. J Neurophysiol 57:1577-1600.

Rotter A (1984) Cholinergic receptors. In: Handbook of chemical neuroanatomy (Bjorklund A, Hokfelt T, Kuhar MJ, eds), pp 273-303. Amsterdam: Elsevier Science.

Sharp FR, Evans K (1982) Regional (14C) 2-deoxyglucose uptake during vibrissae movements evoked by rat motor cortex stimulation. J Comp Neurol 208:255-287.

Simons DJ, Carvell GE (1989) Thalamocortical response transformation in the rat vibrissa/barrel system. J Neurophysiol 61:311-330.

Smaje JC (1976) General anaesthetics and the acetylcholine-sensitivity of cortical neurons. Br J Pharmacol 58:359-366.

Sofroniew MV, Priestley JV, Consolazione A, Eckenstein F, Cuello AC (1985) Cholinergic projections from the midbrain and pons to the thalamus in the rat, identified by combined retrograde tracing and choline acetyltransferase immunohistochemistry. Brain Res 329:213-223.

Timofeeva E, Lavallée P, Arsenault D, Deschênes M (2004) Synthesis of multiwhisker-receptive fields in subcortical stations of the vibrissa system. J Neurophysiol 91:1510-1515.

Vanderwolf CH (1969) Hippocampal electrical activity and voluntary movement in the rat. Electroencephalogr Clin Neurophysiol 26:407-418.

Veinante P, Deschênes M (1999) Single- and multi-whisker channels in the ascending projections from the principal trigeminal nucleus in the rat. J Neurosci 19:5085-5095.

Veinante P, Jacquin MF, Deschênes M (2000) Thalamic projections from the whisker-sensitive regions of the spinal trigeminal complex in the rat. J Comp Neurol 420:233-243.

Vilaro MT, Wiederhold KH, Palacios JM, Mengod G (1992) Muscarinic M2 receptor mRNA expression and receptor binding in cholinergic and noncholinergic cells in the rat brain: a correlative study using in situ hybrid- 
ization histochemistry and receptor autoradiography. Neuroscience 47:367-393.

Waite PM (1973) The responses of cells in the rat thalamus to mechanical movements of the whiskers. J Physiol (Lond) 228:541-561.

Wamsley JK, Lewis MS, Young III WS, Kuhar MJ (1981) Autoradiographic localization of muscarinic cholinergic receptors in rat brainstem. J Neurosci 1:176-191.

Welker WI (1964) Analysis of sniffing of the albino rat. Behav 22:223-244.

Woolf NJ, Butcher LL (1986) Cholinergic systems in the rat brain: III. Projections from the pontomesencephalic tegmentum to the thalamus, tectum, basal ganglia, and basal forebrain. Brain Res Bull 16:603-637.

Woolf NJ, Butcher LL (1989) Cholinergic systems in the rat brain: IV. De- scending projections of the pontomesencephalic tegmentum. Brain Res Bull 23:519-540.

Woolston DC, La Londe JR, Gibson JM (1982) Comparison of response properties of cerebellar- and thalamic-projecting interpolaris neurons. J Neurophysiol 48:160-173.

Yoshida A, Chen K, Moritani M, Yabuta NH, Nagase Y, Takemura M, Shigenaga Y (1997) Organization of the descending projections from the parabrachial nucleus to the trigeminal sensory nuclear complex and spinal dorsal horn in the rat. J Comp Neurol 383:94-111.

Zhang ZW (2004) Maturation of layer V pyramidal neurons in the rat prefrontal cortex: intrinsic properties and synaptic function. J Neurophysiol 91:1171-1182. 